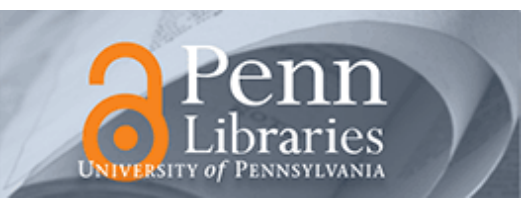

University of Pennsylvania

ScholarlyCommons

Departmental Papers (MEAM)

Department of Mechanical Engineering \&

Applied Mechanics

October 2007

\title{
Dielectric properties of hydrogen-incorporated chemical vapor deposited diamond thin films
}

Chao Liu

Argonne National Laboratory

Xingcheng Xiao

Argonne National Laboratory

Jian Wang

Argonne National Laboratory

Bing Shi

Argonne National Laboratory

Vivekananda P. Adiga

University of Wisconsin

See next page for additional authors

Follow this and additional works at: https://repository.upenn.edu/meam_papers

\section{Recommended Citation}

Liu, Chao; Xiao, Xingcheng; Wang, Jian; Shi, Bing; Adiga, Vivekananda P.; and Carpick, Robert W., "Dielectric properties of hydrogen-incorporated chemical vapor deposited diamond thin films" (2007). Departmental Papers (MEAM). 120.

https://repository.upenn.edu/meam_papers/120

Copyright 2007 American Institute of Physics. This article may be downloaded for personal use only. Any other use requires prior permission of the author and the American Institute of Physics. Reprinted in Journal of Applied Physics, Volume 102, Article 074115 , October 2007,7 pages.

Publisher URL: http://dx.doi.org/10.1063/1.2785874

This paper is posted at ScholarlyCommons. https://repository.upenn.edu/meam_papers/120

For more information, please contact repository@pobox.upenn.edu. 


\title{
Dielectric properties of hydrogen-incorporated chemical vapor deposited diamond thin films
}

\begin{abstract}
Diamond thin films with a broad range of microstructures from a ultrananocrystalline diamond (UNCD) form developed at Argonne National Laboratory to a microcrystalline diamond (MCD) form have been grown with different hydrogen percentages in the $\mathrm{Ar} / \mathrm{CH}_{4}$ gas mixture used in the microwave plasma enhanced chemical vapor deposition (CVD) process. The dielectric properties of the CVD diamond thin films have been studied using impedance and dc measurements on metal-diamond-metal test structures. Close correlations have been observed between the hydrogen content in the bulk of the diamond films, measured by elastic recoil detection (ERD), and their electrical conductivity and capacitance-frequency ( $C$ f) behaviors. Addition of hydrogen gas in the $\mathrm{Ar} / \mathrm{CH}_{4}$ gas mixture used to grow the diamond films appears to have two main effects depending on the film microstructure, namely, (a) in the UNCD films, hydrogen incorporates into the atomically abrupt grain boundaries satisfying $s p^{2}$ carbon dangling bonds, resulting in increased resistivity, and (b) in MCD, atomic hydrogen produced in the plasma etches preferentially the graphitic phase codepositing with the diamond phase, resulting in the statistical survival and growth of large diamond grains and dominance of the diamond phase, and thus having significant impact on the dielectric properties of these films.
\end{abstract}

\section{Keywords}

chemical vapour deposition, diamond, dielectric properties, dielectric thin films, grain boundaries, nanostructured materials, nuclear chemical analysis

\section{Comments}

Copyright 2007 American Institute of Physics. This article may be downloaded for personal use only. Any other use requires prior permission of the author and the American Institute of Physics. Reprinted in Journal of Applied Physics, Volume 102, Article 074115, October 2007, 7 pages.

Publisher URL: http://dx.doi.org/10.1063/1.2785874

\section{Author(s)}

Chao Liu, Xingcheng Xiao, Jian Wang, Bing Shi, Vivekananda P. Adiga, and Robert W. Carpick 


\title{
Dielectric properties of hydrogen-incorporated chemical vapor deposited diamond thin films
}

\author{
Chao Liu, ${ }^{\text {a) }}$ Xingcheng Xiao, Jian Wang, and Bing Shi \\ Materials Science Division, Argonne National Laboratory, Argonne, Illinois 60439, USA \\ Vivekananda P. Adiga \\ Materials Science Program, University of Wisconsin-Madison, Madison, Wisconsin 53706, USA \\ Robert W. Carpick \\ Department of Mechanical Engineering and Applied Mechanics, University of Pennsylvania, \\ Philadelphia, Pennsylvania 19104, USA \\ John A. Carlisle and Orlando Auciello \\ Materials Science Division, Argonne National Laboratory, Argonne, Illinois 60439, USA \\ and Center for Nanoscale Materials, Argonne National Laboratory, Argonne, Illinois 60439, USA
}

(Received 28 November 2006; accepted 8 August 2007; published online 11 October 2007)

\begin{abstract}
Diamond thin films with a broad range of microstructures from a ultrananocrystalline diamond (UNCD) form developed at Argonne National Laboratory to a microcrystalline diamond (MCD) form have been grown with different hydrogen percentages in the $\mathrm{Ar} / \mathrm{CH}_{4}$ gas mixture used in the microwave plasma enhanced chemical vapor deposition (CVD) process. The dielectric properties of the CVD diamond thin films have been studied using impedance and dc measurements on metal-diamond-metal test structures. Close correlations have been observed between the hydrogen content in the bulk of the diamond films, measured by elastic recoil detection (ERD), and their electrical conductivity and capacitance-frequency $(C-f)$ behaviors. Addition of hydrogen gas in the $\mathrm{Ar} / \mathrm{CH}_{4}$ gas mixture used to grow the diamond films appears to have two main effects depending on the film microstructure, namely, (a) in the UNCD films, hydrogen incorporates into the atomically abrupt grain boundaries satisfying $s p^{2}$ carbon dangling bonds, resulting in increased resistivity, and (b) in MCD, atomic hydrogen produced in the plasma etches preferentially the graphitic phase codepositing with the diamond phase, resulting in the statistical survival and growth of large diamond grains and dominance of the diamond phase, and thus having significant impact on the dielectric properties of these films. (C) 2007 American Institute of Physics.
\end{abstract}

[DOI: $10.1063 / 1.2785874]$

\section{INTRODUCTION}

The ultrananocrystalline diamond (UNCD) material developed in thin film form at Argonne National Laboratory ${ }^{1}$ is unique among the family of polycrystalline diamond thin films, due to its nanocrystalline structure. UNCD thin film is deposited using microwave plasma chemical vapor deposition (MPCVD) with argon-rich/methane plasmas that produce a film nanostructure consisting of $s p^{3}$-bonded diamond grains with 3-5 nm grain size and atomically abrupt $(0.4 \mathrm{~nm}$ wide) grain boundaries with a mixture of $s p^{1}, s p^{2}$, and $s p^{3}$ carbon bonds. ${ }^{2}$ Extensive experimental studies have revealed superb properties of UNCD including high hardness, high fracture strength, and extreme smooth surface. ${ }^{3-5}$ It has also been reported that the electronic properties of UNCD thin films are strongly affected by hydrogen and/or nitrogen atoms incorporated in the film. 6,7

Our group has also demonstrated that UNCD films exhibit a unique combination of superior mechanical and tribological properties that make them ideally suited to produce a new generation of high performance microelectromechanical system (MEMS) and nanoelectromechanical system (NEMS)

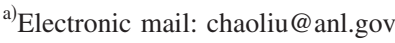

devices. A specific example pertinent to this paper is that UNCD thin film can be used as a low-trap dielectric material in rf MEMS capacitive switches, due to its outstanding properties mentioned above as well as its intrinsically hydrophobic and low energy surface yielding fast response and long lifetime operation of the switch. Moreover, by using UNCD as a dielectric layer with controlled leakage current can minimize or eliminate the charging problem currently observed in the oxide dielectric layers used in $\mathrm{rf}$ capacitive switches. ${ }^{4}$ In order to utilize CVD diamond thin film in such applications, its dielectric characteristics need to be investigated.

There is substantial literature on the dielectric properties of diamond. However, most of the published papers focused on natural diamond, ${ }^{8,9}$ single crystal homoepitaxial diamond, ${ }^{10}$ and microcrystalline diamond thin films. ${ }^{11-14}$ In most reports on characterization of the dielectric properties of diamond layers, capacitive measurements of metaldiamond-metal structure were performed using freestanding diamond films or films grown on substrate such as silicon. In general, dielectric properties of diamond thin film (dielectric constant and loss tangent) were found to be frequency dependent, and it is widely accepted that dielectric loss is closely related to the quality of the diamond film, specifically the defects in the diamond layer. Higher loss tangents were 
observed for lower-quality diamond films. ${ }^{12,15}$ Also, dielectric constant has been observed to be dependent on the resistivity of the diamond film as well, i.e., the higher the resistivity, the lower the dielectric constant, for microcrystalline CVD diamond films. ${ }^{12}$ At low frequencies, a Hill-type hopping conduction mechanism was proposed as a predominant factor for the frequency dependence of the permittivity and loss tangent. On the other hand, combination of multiple charge transport and relaxation processes makes the high frequency response more complicated. ${ }^{15-17}$ However, much less work has addressed the dielectric characteristics of nanocrystalline diamond and definitely no systematic studies were performed until now on the dielectric properties of the UNCD films. ${ }^{13}$

In this work, current-voltage $(I-V)$ and capacitancefrequency $(C-f)$ measurements were performed on metaldiamond-metal capacitor test structures. CVD diamond films with various hydrogen-incorporation levels were studied. Properties of diamond thin film, including relative permittivity (dielectric constant), loss factor (loss tangent), phase purity, bulk hydrogen concentration, and resistivity, were examined for each sample. Raman spectroscopy, scanning electron microscopy (SEM), and atomic force microscopy (AFM) were applied to examine the bonding characteristics and morphology of diamond samples. Elastic recoil detection (ERD) measurement was performed to determine the hydrogen content in the diamond films. ERD has been accepted by the material research community as one of the most accurate and reliable methods to quantitatively analyze light elements and isotopes, such as hydrogen and deuterium, in carbonbased materials including polymer, diamond, and diamond like carbon. ${ }^{18-22}$

The objectives of this work were to study the dielectric properties of CVD diamond thin films, and to correlate the dielectric properties with the hydrogen-incorporation level, all this with a view at application of CVD diamond thin film as a dielectric layer for rf MEMS switches.

\section{EXPERIMENTAL}

Hydrogen-incorporated CVD diamond thin films were synthesized in a MPCVD reactor (iPlas). Prior to the thin film growth, a $100 \mathrm{~nm}$ thick tungsten layer was sputtered onto $\mathrm{SiO}_{2} / \mathrm{Si}$ (thermal oxide $1 \mu \mathrm{m}$ thick) substrate as the bottom electrode. The reasons of using refractory metal such as tungsten as bottom electrode are as follows: (a) it can survive the elevated temperature during the thin film deposition and (b) prior work by our group has demonstrated that the $\mathrm{W}$ layer acts as a very efficient nucleation promotion layer to achieve much smoother and denser UNCD films. The surface of the tungsten layer was then seeded in ultrasonic bath with nanocrystalline diamond powder $(5 \mathrm{~nm}$ in diameter averagely) suspended in methanol. After sequential rinse with isopropanol, acetone, and methanol, the substrate was blow dry with nitrogen. The CVD diamond films were deposited at $\sim 800{ }^{\circ} \mathrm{C}$ on substrates located on a molybdenum-coated graphite holder resistively heated from underneath. Argon and methane (2\% in volume) gas mixture, with addition of hydrogen gas, was used as feedstock. The

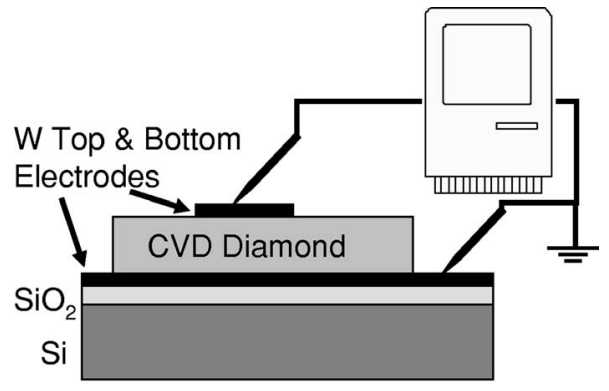

FIG. 1. Schematic diagram of the measurement setup and cross-sectional view of a metal-diamond-metal test structure.

volume percentage of hydrogen in the gas mixture was systematically changed to produce a set of samples with controlled amounts of hydrogen incorporation. $\mathrm{Ar} / \mathrm{CH}_{4} / \mathrm{H}_{2}$ plasmas were produced by a $1 \mathrm{~kW}$ microwave plasma enhanced chemical vapor deposition system to grow the UNCD and MCD films with 2-3 $\mu \mathrm{m}$ thickness. Following diamond film deposition, a patterned $\mathrm{W}$ layer was deposited onto the diamond top surface via sputtering through a shadow mask to define $\mathrm{W}$ dots with $250 \mu \mathrm{m}$ in diameter so as to produce the metal-diamond-metal capacitor structure required for the measurements. In order to assure full coverage of the rougher surface of the as grown MCD thin film, a relatively thick $(\sim 300 \mathrm{~nm}) \mathrm{W}$ layer was deposited at this time.

The surface morphology of diamond films as well as their thicknesses were examined by top view and crosssectional SEM. The surface roughness was measured using AFM. ERD measurements were carried out using a $3 \mathrm{MeV}$ ${ }^{4} \mathrm{He}^{++}$ion beam with a current of $10 \mathrm{nA}$ and a grazing angle of $15^{\circ}$ with respect to the film surface in a high vacuum environment. An aluminum stopping foil was used to filter out the reflected helium ions, allowing the recoiled hydrogen to be measured by a silicon detector. A polystyrene (PS) film known to have a uniformly distributed hydrogen fraction of $50 \%$ was also measured as a standard to calibrate the hydrogen counts. A depth profile with a spatial resolution of $\sim 70 \mathrm{~nm}$ has been achieved by this instrument. Impedance and $I-V$ measurements were carried out on a probe station connected to a HP4192A impedance analyzer and a Keithley 237 voltage source (Fig. 1) at room temperature. The bonding structure of the CVD diamond thin films was determined via Raman analysis using a Renishaw Raman spectroscope with a $\mathrm{HeNe}$ laser at $\lambda=632.8 \mathrm{~nm}$.

\section{RESULTS AND DISCUSSION}

\section{A. SEM, AFM, and Raman characterizations}

Figure 2 shows the surface morphology of CVD diamond thin films grown with various percentage of hydrogen in the $\mathrm{Ar} / \mathrm{CH}_{4}$ gas mixture. All diamond films exhibit a dense microstructure at low magnification, although higher magnification SEM pictures show a change from the ultrananocrystalline structure for films grown with $0 \% \mathrm{H}_{2}$ in the plasma [Fig. 2(a)] to a nanocrystalline transition film at $5 \% \mathrm{H}_{2}$ [Fig. 2(b)]. Figure 2(c) exhibits a combination of nanocrystalline and microcrystalline structures for diamond thin films grown with $10 \% \mathrm{H}_{2}$ in the gas phase. Surface 


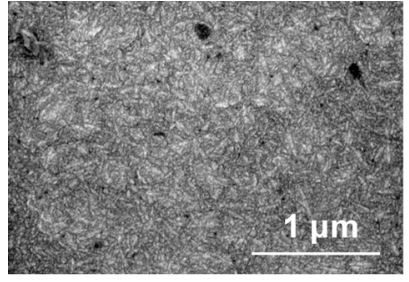

(a)

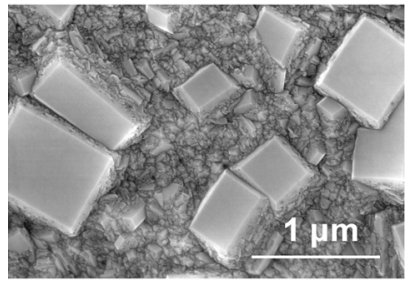

(c)

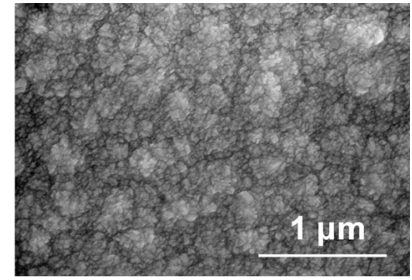

(b)

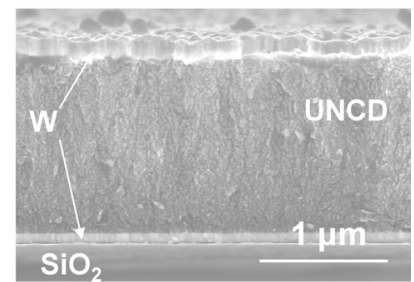

(d)
FIG. 2. SEM photographs of surfaces of diamond thin films deposited with Ar-rich/ $\mathrm{CH}_{4}$ microwave plasmas with (a) $0 \%$, (b) $5 \%$, and (c) $10 \%$ hydrogen in the $\mathrm{Ar} / \mathrm{CH}_{4}$ gas mixture, respectively. Figure (d) illustrates crosssectional view of the tungsten-diamond-tungsten test structure on $\mathrm{SiO}_{2} / \mathrm{Si}$ substrate.

roughness measurements also confirm the morphology change from a ultrananocrystalline structure $(\sim 20 \mathrm{~nm} \mathrm{rms})$ to a mixture of microcrystalline and nanocrystalline structures $(\sim 250 \mathrm{~nm} \mathrm{rms})$.

Raman spectra of corresponding diamond thin films are shown in Fig. 3. Raman spectra corresponding to CVD diamond films grown with $0 \%, 1 \%$, and $2 \%$ hydrogen show typical ultrananocrystalline diamond features with two broad peaks at 1330 and $1580 \mathrm{~cm}^{-1}$. These two dominant features arise from the $s p^{2}$ dominant bonding in the grain boundaries of the films, where the grain boundary occupies a relatively large $(\sim 10 \%)$ volume fraction. More detailed interpretation of the CVD diamond thin film Raman spectrum can be found in a recent publication. ${ }^{23}$ When the hydrogen concentration in the plasma increases further beyond 5\%, a microcrystalline diamond characteristic peak at $1332 \mathrm{~cm}^{-1}$ starts to appear. Meanwhile, the slight decrease of peak intensity at 1150 and $1440 \mathrm{~cm}^{-1}$, which are attributed to the transpolyacetylene along the grain boundary, indicates the de-

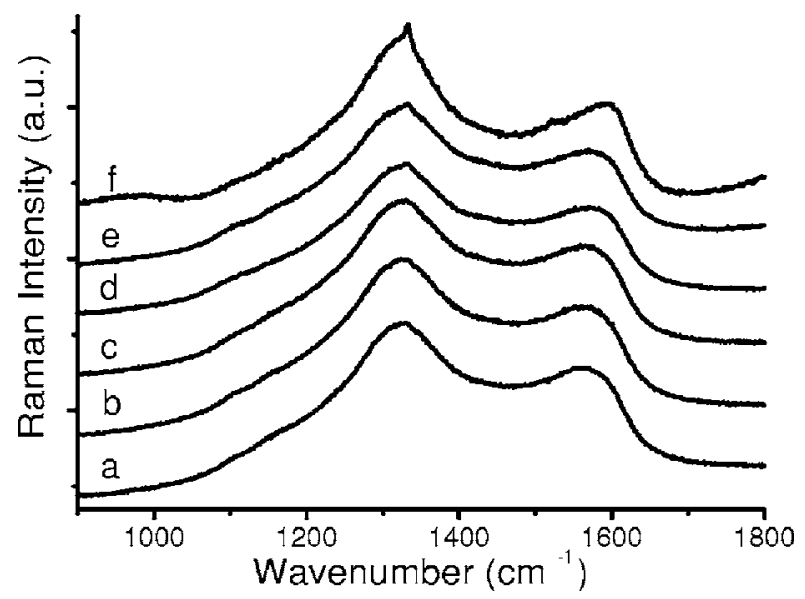

FIG. 3. Raman spectra of diamond thin films deposited in microwave plasmas with (a) $0 \%$, (b) $1 \%$, (c) $2 \%$, (d) $3 \%$, (e) $5 \%$, and (f) $10 \%$ hydrogen in the $\mathrm{Ar} / \mathrm{CH}_{4}$ gas mixture, respectively.

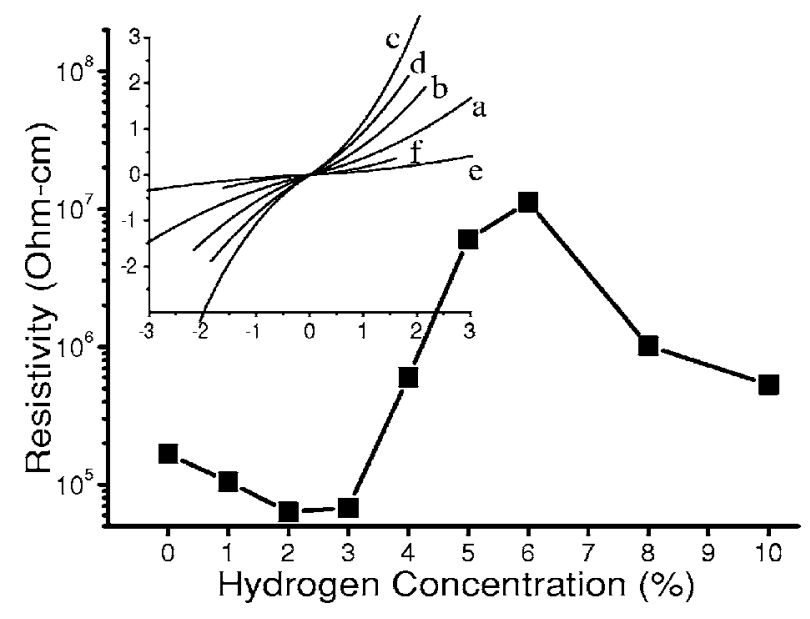

FIG. 4. Schematic plotting of CVD diamond thin film resistivity as a function of hydrogen percentage in the gas mixture during film growth. Inset: $I-E$ curves measured from test structure with diamond thin films deposited in microwave plasmas with (a) $0 \%$, (b) $1 \%$, (c) $2 \%$, (d) $3 \%$, (e) $5 \%$, and (f) $10 \%$ hydrogen in the $\mathrm{Ar} / \mathrm{CH}_{4}$ gas mixture $[\mathrm{X}$ axis: electric field $(\mathrm{V} / \mu \mathrm{m}) ; Y$ axis: current $\left.\left(10^{-4} \mathrm{~A}\right)\right]$.

crease of relative fraction of grain boundaries in the film. Combining the SEM photographs and Raman spectra, it is clear that, with increasing hydrogen percentage in the gas feedstock, the microscopic structure of the diamond thin films changes from ultrananocrystalline diamond to a mixture of ultrananocrystalline, nanocrystalline, and microcrystalline diamond.

\section{B. $I-V$ and ERD measurements}

The polycrystalline nature of CVD diamond thin films makes its charge transport behavior much more complicated. It has been generally accepted that the grain boundary is a predominant factor on the charge transport through polycrystalline diamond, even though its mass and volume percentage are fairly small. During the study of $I-V$ behavior of CVD diamond thin films, a simplification is made assuming that the possible Schottky effect between the diamond layer and metal electrode is small and can be ignored.

The resistivity of CVD diamond thin films is calculated using $I-V$ measurement data at low electric field as

$$
\rho=\frac{V A}{I d},
$$

where $\rho$ is the resistivity, $V$ is the applied voltage across the top and bottom electrodes, $A$ is the area of the top electrode, $I$ is the current measured flowing across the diamond thin film from one electrode to another, and $d$ is the thickness of the diamond film. The result is plotted in Fig. 4, accompanied by an insertion of typical $I-E$ curves measured from several test devices. All $I-E$ curves have similar shape but different curvatures and slopes. For every sample, the $I-E$ relationship is quasilinear at low electric field, and becomes more nonlinear when the electric field increases. This nonOhmic behavior at high electric field may be attributed to the effects of thermal excitation (Joule heating), carrier density enhancement, and conductivity increase at high current levels. ${ }^{24}$ The resistivity data plotted in Fig. 4 are obtained 

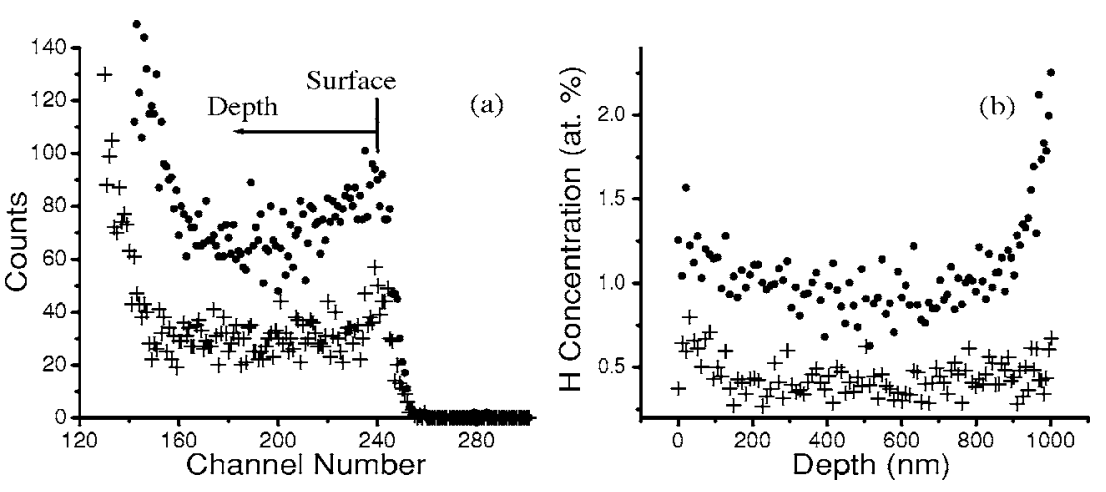

FIG. 5. Hydrogen ERD spectra (a) and hydrogen depth profile (b) for diamond thin film samples deposited in microwave plasmas with $2 \%$ (dots) and 6\% (crosses) hydrogen in the $\mathrm{Ar} / \mathrm{CH}_{4}$ gas mixture, respectively. from the low electric field region of the $I-E$ curves in order to minimize the effects described above. It can be observed that, as hydrogen percentage in the gas feedstock increases, the resistivity of diamond thin films firstly decreases slightly, and then increases more than two orders of magnitude from $3 \%$ to $6 \%$ hydrogen concentration in the source gas during deposition process. Then, the resistivity decreases as hydrogen percentage further increases. It should be noticed that the dip in the resistivity versus $\mathrm{H}_{2}$ concentration curve at low $\mathrm{H}_{2}$ percentage $(1 \%-3 \%)$ is not as significant as it appears because the curve is plotted in logarithmic scale.

In order to examine the role of hydrogen in the diamond thin film dielectric properties, accurate hydrogen concentration in the diamond thin film should be provided. ERD spectra were obtained for diamond thin films grown in $\mathrm{Ar} / \mathrm{CH}_{4}$ plasmas with additional hydrogen concentrations of $0 \%, 1 \%$, $2 \%, 4 \%, 5 \%, 6 \%$, and $10 \%$, respectively. For better illustration, Fig. 5(a) shows only two well-separated ERD spectra corresponding to diamond thin films grown in $\mathrm{Ar} / \mathrm{CH}_{4}$ plasmas with additional $2 \%$ and $6 \%$ hydrogen in the gas mixture, respectively. The rest of the spectra have similar shape and fall in between these two. It has been observed that, for each spectrum, there is a peak which corresponds to the surface concentration of the hydrogen species. The width of this peak depends on the energy resolution of the instrument. A low energy tail into the bulk of each sample indicates that there is a detectable amount of hydrogen content in the bulk. By comparing the ERD spectrum of diamond sample with that of the PS film standard, the hydrogen depth profile of each sample can be plotted. Figure 5(b) shows hydrogen depth profile calculated from the corresponding ERD spectra in Fig. 5(a).

The slightly higher hydrogen concentration at the surface illustrated in Fig. 5(b) can be explained by either hydrogen termination of diamond surface $e^{19,22}$ or surface adsorption of hydrogen-containing species, ${ }^{25}$ for instance, water molecules. The increase of the hydrogen concentration after 800 $\mathrm{nm}$ is due to the reflected He atoms at low energy during the ERD measurement and should be disregarded. Therefore, in order to accurately estimate the bulk concentration of hydrogen content in the diamond thin film, data points in the hydrogen depth profile are averaged from 200 to $800 \mathrm{~nm}$ for each sample. The calculated bulk hydrogen concentrations for the diamond samples are plotted in Fig. 6 curve (a) as a function of hydrogen concentration in the plasma during thin film deposition. The values of bulk hydrogen concentration measured from this set of diamond samples are comparable with those reported in the literature. ${ }^{20,21,26}$

For better understanding of the effect of hydrogen concentration in CVD diamond film on its electrical conductivity behavior, Fig. 4 is replotted in Fig. 6 as curve (b). By taking reciprocal of the original resistivity values in Fig. 4, this curve illustrates the conductivity of CVD diamond thin films as a function of hydrogen concentration in the plasma. It can be observed that these two curves follow each other in a very close manner, which indicates that the hydrogen concentration in the diamond film has a fundamental impact on its electrical conductivity. The insert in Fig. 6 shows the electrical conductivity of diamond films ( $Y$ axis) as a function of bulk hydrogen concentration ( $X$ axis). The quasilinear shape of the data curve also provides another indication of the close link between the hydrogen concentration in diamond films and their electrical conductivity.

It has been reported that, besides defective regions in diamond grains, hydrogen is mainly incorporated in the grain boundaries, forming $\mathrm{C}-\mathrm{H}$ bonds such as hydrogenterminated carbon dangling bonds. ${ }^{27-29}$ When the hydrogen concentration in the gas mixture is below $2 \%$, the microstructure of deposited diamond film is dominant by nanocrystalline grains. Having relatively largest amount of grain boundaries $(\sim 10 \%$ in volume $)$, hydrogen incorporations in

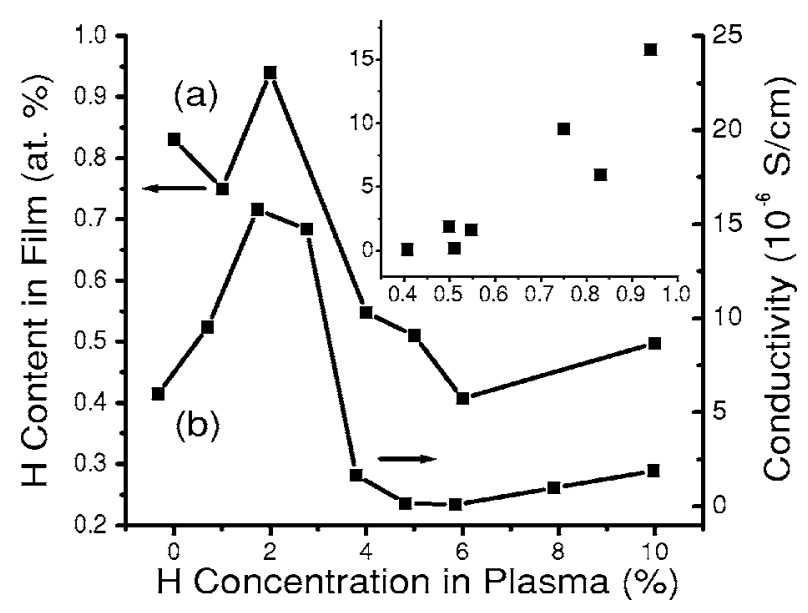

FIG. 6. (a) Bulk hydrogen concentrations in diamond thin films as a function of hydrogen concentration in plasma. (b) A replot of Fig. 4 in linear scale by taking a reciprocal of resistivity data. Inset: A quasilinear relationship between the electrical conductivity of diamond films ( $Y$ axis) and their bulk hydrogen concentrations ( $X$ axis). 

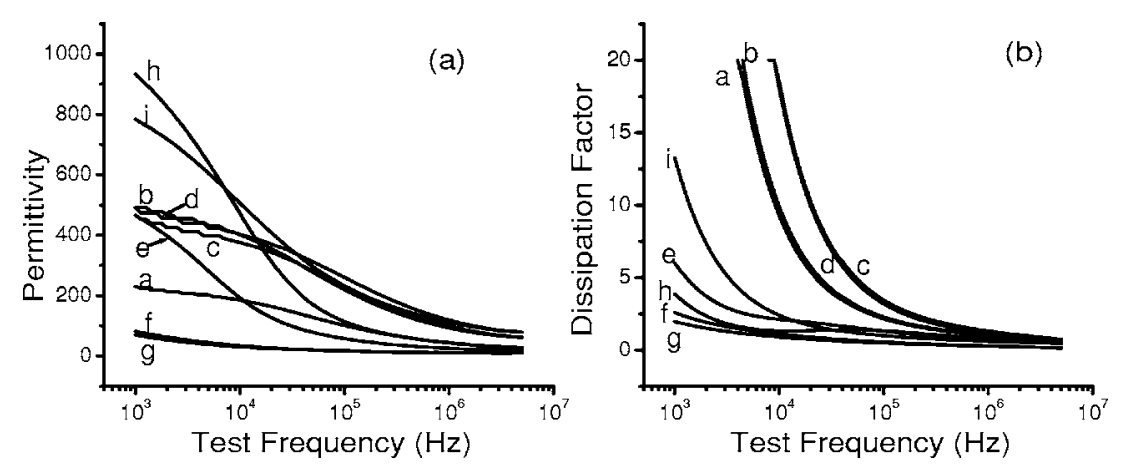

FIG. 7. Calculated permittivity (a) and measured dissipation factor (b) as a function of test frequency for diamond thin films deposited in microwave plasmas with (a) $0 \%$, (b) $1 \%$, (c) $2 \%$, (d) $3 \%$, (e) $4 \%$, (f) $5 \%$, (g) $6 \%$, (h) $8 \%$, and (i) $10 \%$ hydrogen in the $\mathrm{Ar} / \mathrm{CH}_{4}$ gas mixture, respectively. these diamond films are enhanced, resulting in the highest bulk hydrogen concentrations in these films. On the other hand, the structure of the diamond films changes from ultrananocrystlline to mixture of nanocrystalline/ microcrystalline diamond with increasing hydrogen concentrations in the plasma in the range of 4\%-10\%. Smaller amount of grain boundaries exist in diamond films with nanocrystalline (up to a few hundred nanometers in diameter) and microcrystalline ( $>1 \mu \mathrm{m}$ in diameter) structures as opposed to UNCD films that exhibit 2-5 nm diameter grains. This explains the smaller amount of hydrogen detected by ERD in NCD and MCD films, comparing with the hydrogen content in the UNCD films. In the discussion presented above, an assumption has been made that hydrogen atoms incorporate into the diamond thin film grain boundaries at a constant level regardless the hydrogen concentration in the plasma during the deposition process, i.e., the absolute amount of hydrogen content in the CVD diamond thin film is proportional to the grain boundary volume in the film. It is known, from the literature, that hydrogen incorporation into CVD diamond films depends strongly on the deposition conditions, among which the substrate temperature is a key parameter. ${ }^{30}$ Giving that the CVD diamond thin films discussed in this paper were deposited at the same substrate temperature $\left(\sim 800^{\circ} \mathrm{C}\right)$, the above assumption is reasonable and valid.

Considering that grain boundaries in the diamond films discussed here contain high density of defects, impurities, and conductive carbon species such as $s p^{1}$ - and $s p^{2}$-bonded carbon atoms, it is understandable that they have a dominant effect on electron transport in these polycrystalline diamond films. The nondiamond carbon species and carbon dangling bonds in the grain boundaries introduce localized states in the diamond band gap, contributing to electron transport in diamond thin films. Thus, the electrical conductivity of CVD diamond thin films can be correlated with the volume percentage of the grain boundary phase in the film. When hydrogen gas is added into the plasma, more atomic hydrogen, which is a strong etcher of graphitic carbon, is generated, resulting in a change of volume ratio of diamond phase to nondiamond phase. Therefore, a close relationship between the electrical conductivity of the diamond films and their bulk hydrogen concentration shown in Fig. 6 can be correlated to the grain boundaries present in the films.

A conclusion drawn from the above discussions is that the amount of grain boundary present in the diamond thin film discussed in this paper changes as a function of hydro- gen gas, which impacts the plasma chemistry during deposition, added to the $\mathrm{Ar} / \mathrm{CH}_{4}$ gas mixture. Hydrogen atoms are preferentially incorporated into the grain boundaries to satisfy the carbon dangling bonds, resulting in a change of bulk hydrogen concentration in the film as a function of hydrogen concentration in the gas mixture. On the other hand, the electrical conductivity of the CVD diamond thin film is dominated by its grain boundary. Therefore the close relationship between the electrical conductivity and bulk hydrogen concentration of the diamond film observed in Fig. 6 is reasonable, because they can both be correlated with the grain boundary in the film, which is in turn dependent on the hydrogen concentration in the gas mixture during the CVD process.

\section{C. $\boldsymbol{C}$-f measurement}

The metal-diamond-metal test structure fabricated on the $\mathrm{SiO}_{2} / \mathrm{Si}$ substrate forms a parallel plate capacitor. In an ideal case, the relative permittivity $\left(\varepsilon_{r}\right)$ of a dielectric material in the middle of two parallel electrodes can be calculated as

$$
\varepsilon_{r}=\frac{C d}{A \varepsilon_{0}},
$$

where $C$ is the measured capacitance, $d$ is the distance between two parallel electrodes, which is equivalent to the thickness of the dielectric thin film, $\varepsilon_{0}$ is the permittivity of free space, and $A$ is the area of the electrodes. However, the real metal-diamond-metal test device deviates from the ideal parallel plate capacitor in a couple of aspects. Firstly, the geometrical structure of the test device is not ideal because only the top tungsten metal dot is patterned with known diameter. The bottom electrode in the samples used in the work reported here is a large planar $\mathrm{W}$ layer instead of being patterned as the top electrode. Secondly, the effective area underneath the top electrode is larger than the size of the metal dot due to the surface roughness of the diamond thin films. Thus, the calculated relative permittivity using Eq. (2) should be an effective value. However, the effects discussed above shall be small and negligible for relative comparison among the diamond samples studied in this work.

The frequency responses of hydrogen-incorporated diamond capacitors were obtained using an impedance analyzer with parallel mode at room temperature. The effective relative permittivities and dissipation factors of the CVD diamond thin films, which were produced with various hydrogen concentrations in the gas mixture during deposition, are 
plotted as functions of test frequency in Fig. 7. It can be observed that the frequency response curves fall into three categories:

(a) The first group [Fig. 7(a), curves a-d], corresponding to diamond films grown with $0 \%-3 \%$ hydrogen concentration in the plasma, exhibits moderate value of relative permittivity at low frequencies, and their permittivity values decrease gradually as a function of increasing test frequency.

(b) The second group [Fig. 7(a), curves f and g], including diamond films produced with $5 \%$ and $6 \%$ hydrogen concentrations in the plasma, presents relatively low and almost constant permittivities throughout the entire test frequency range.

(c) The third group [Fig. 7(a), curves e, h, and i], including diamond films grown with $4 \%, 8 \%$, and $10 \%$ hydrogen in the plasma, starts with large relative permittivity values at low frequency and drops drastically with increasing test frequency.

Figure 7(b) presents the frequency dependence of the dissipation factor correlating with the permittivity curves shown in Fig. 7(a). Also, having a close correlation with their permittivity and resistivity characteristics, the samples exhibit high (Fig. 7(b), curves a-d), moderate (curves e, i, and h), and low (curves f and g) losses. Considering the samples' resistivity values illustrated in Fig. 4, it is obvious that these three groups correlate with the three categories of resistivity: low, high, and moderate resistivities, respectively.

Material's content and structural regularities (or irregularities) are two major factors influencing macroscopic electrical properties. ${ }^{12}$ Hence, the dielectric properties of the CVD diamond thin films studied here should be affected by their polycrystalline nature as well as the presence of nondiamond phase in the grain boundaries. From the above discussion, it can be concluded that the dielectric constant and loss factor of the hydrogen-incorporated CVD diamond samples follow their resistivity behavior very closely. Higher dissipation factor and lower resistivity of diamond films grown with low hydrogen percentage $(0 \%-3 \%)$ in the plasma reflect the presence of more nondiamond $\left(s p^{1}\right.$ and $s p^{2}$ ) carbon phases in the grain boundaries of the films. On the other hand, diamond thin films produced with $5 \%$ and $6 \%$ hydrogen in the plasma exhibit better quality in terms of less nondiamond carbon and low defect density. This also reflects the role of conductive (leakage) current playing in the capacitive measurement. It is well known that, besides relaxation process, charge transport (including free charge and hopping conductivities) has a major contribution to the experimental loss tangent of dielectric materials. Therefore, a low-resistive dielectric material, which gives higher leakage current, exhibits higher loss during capacitive measurement.

The relative permittivity for natural diamond is 5.7 . $^{1}$ Various and strongly sample-dependent permittivity values, for instance, $4.2-8,{ }^{12} 0.95-8.13,{ }^{13}$ and 11.1 and $19.8,{ }^{31}$ were reported for CVD polycrystalline diamond thin films. The relative permittivity values measured from $5 \%$ and $6 \%$ hydrogen samples are comparable with what have been re- ported in the literatures. Higher values measured from other samples indicate a low quality, lossy, and high leakage current nature of those films.

It can be observed from Fig. 7(b) that at low frequencies, the dissipation factor is large for the low- and moderateresistivity samples. This may weaken the validity of those data. However, the measurement data in the high-loss range are still useful in the senses of comparison among samples, illustrating the trend of frequency dependence, and indicating the level of dielectric loss as well as the quality of diamond thin films.

It is necessary to clarify that in the discussion of frequency response of metal-diamond-metal capacitor, the diamond thin film is treated as a homogeneous material with uniform microscopic structure. Although it is a necessary simplification for macroscopic study of a material, however, the polycrystalline structure of CVD diamond film consisting of diamond grains and grain boundaries has a fundamental impact on its dielectric properties. Thus, more effort on studying the two-domain nature of CVD diamond thin film shall be carried out

\section{CONCLUSIONS}

In this work, dielectric properties of CVD diamond thin films deposited with various hydrogen concentrations in the source gas were studied. The resistivity of CVD diamond films exhibits a close relationship with their bulk hydrogen concentration, which is affected by the hydrogen percentage in the gas mixture during the CVD process. Moreover, the capacitance-frequency response and dielectric loss of the CVD diamond films studied here exhibit a strong dependence on their electrical conductivities. Hydrogen incorporation in the grain boundaries of the CVD diamond thin films has a significant impact on their permittivity and resistivity values, via saturating carbon dangling bonds at the grain boundaries and lowering the defect density, as well as changing the microscopic structure of the film. Microstructural changes may result from selective etching of nondiamond phase by atomic hydrogen, resulting in the microcrystalline growth regime. In addition to the effect of the microstructural changes, change of volume ratio of diamond phase and nondiamond phase in the film, is a major contributor to the dramatic changes in the dielectric properties of CVD diamond thin films studied in this work.

\section{ACKNOWLEDGMENTS}

The submitted manuscript has been created by UChicago Argonne, LLC, Operator of Argonne National Laboratory ("Argonne"). Argonne, a U.S. Department of Energy Office of Science laboratory, is operated under Contract No. DEAC02-06CH11357. The authors thank Professor Russell J. Composto at University of Pennsylvania for useful discussions and assistance with the ERD measurements.

${ }^{1}$ D. M. Gruen, Annu. Rev. Mater. Sci. 29, 211 (1999).

${ }^{2}$ D. M. Gruen, S. Liu, A. R. Krauss, J. Lou, and X. Pan, Appl. Phys. Lett. 64, 1502 (1994)

${ }^{3}$ H. D. Espinosa, B. Peng, B. C. Prorok, N. Moldovan, O. Auciello, J. A. Carlisle, D. M. Gruen, and D. C. Mancini, J. Appl. Phys. 94, 6076 (2003). 
${ }^{4}$ O. Auciello, J. Birrell, J. Carlisle, J. Gerbi, X. Xiao, B. Peng, and H. Espinosa, J. Phys.: Condens. Matter 16, 539 (2004).

${ }^{5}$ X. Xiao, J. Wang, C. Liu, J. A. Carlisle, B. Mech, R. Greenberg, D. Guven, R. Freda, M. S. Humayun, J. Weiland, and O. Auciello, J. Biomed. Mater. Res., Part B: Appl. Biomater. 77B, 273 (2006).

${ }^{6}$ S. Jiao, A. Sumant, M. A. Kirk, D. M. Gruen, A. R. Krauss, and O. Auciello, J. Appl. Phys. 90, 118 (2001).

S. Bhattacharyya, O. Auciello, J. Birrell, J. A. Carlisle, L. A. Curtiss, A. N. Goyette, D. M. Gruen, A. R. Krauss, J. Schlueter, A. Sumant, and P. Zapol, Appl. Phys. Lett. 79, 1441 (2001).

${ }^{8}$ G. H. Glover, Solid-State Electron. 16, 973 (1973).

${ }^{9}$ M. W. Geis, J. A. Gregory, and B. B. Pate, IEEE Trans. Electron Devices 38, 619 (1991).

${ }^{10}$ R. Ramesham, P. E. Pehrsson, T. I. Smith, and M. F. Rose, J. Mater. Sci.: Mater. Electron. 8, 69 (1997).

${ }^{11}$ J. A. von Windheim, V. Venkatesan, D. M. Mlta, and K. Das, Diamond Relat. Mater. 2, 841 (1993).

${ }^{12}$ S. Heidger, S. Fries-Carr, J. Weimer, B. Jordan, and R. Wu, Proceedings of the IEEE 1998 National Aerospace and Electronics Conference (NAECON 1998), pp. 247-254.

${ }^{13}$ H. Ye, Q. S. Chang, and P. Hing, J. Phys. D 33, L148 (2000).

${ }^{14}$ G. Conte, M. C. Rossi, F. Spaziani, and R. Arcangeli, Diamond Relat. Mater. 14, 570 (2005).

${ }^{15}$ F. X. Lu, H. D. Zhang, Y. M. Tong, J. X. Yang, C. M. Li, G. C. Chen, and W. Z. Tang, Diamond Relat. Mater. 13, 1714 (2004).

${ }^{16}$ A. Ibarra, M. González, R. Vila, and J. Mollá, Diamond Relat. Mater. 6, 856 (1997).

${ }^{17}$ J. Mollá, A. Ibarra, and C. Maffiotte, Diamond Relat. Mater. 9, 1071 (2000).
${ }^{18}$ R. J. Composto, R. M. Walters, and J. Genzer, Mater. Sci. Eng., R. 38, 107 (2002).

${ }^{19}$ H. Yagi, K. Tanida, K. Nishimura, A. Hatta, T. Ito, and A. Hiraki, Jpn. J. Appl. Phys., Part 2 34, L577 (1995).

${ }^{20}$ D. K. Avasthi, Vacuum 47, 1249 (1996).

${ }^{21}$ A. Kimura, Y. Nakatani, K. Yamada, and T. Suzuki, Diamond Relat. Mater. 8, 37 (1999).

${ }^{22}$ K. Kimura, K. Nakajima, S. Yamanaka, M. Hasegawa, and H. Okushi, Nucl. Instrum. Methods Phys. Res. B 190, 689 (2002).

${ }^{23}$ J. Birrell, J. E. Gerbi, O. Auciello, J. M. Gibson, J. Johnson, and J. A. Carlisle, Diamond Relat. Mater. 14, 86 (2005).

${ }^{24}$ J. Davison, W. Kang, K. Holms, A. Wisitsora-At, P. Taylor, V. Pulugurta, R. Venkatasubramanian, and F. Wells, Diamond Relat. Mater. 10, 1736 (2001).

${ }^{25}$ C. A. Zorman, G. T. Mearini, and R. W. Hoffman, Diamond Relat. Mater. 9, 1518 (2000).

${ }^{26}$ E. Titus, N. Ali, G. Cabral, J. C. Madaleno, V. F. Neto, J. Gracio, P. R. Babu, A. K. Siker, T. I. Okpalugo, and D. S. Misra, Thin Solid Films 515, 201 (2006).

${ }^{27}$ B. Dischler, C. Wild, W. Muller-Sebert, and P. Koidl, Physica B 185, 217 (1993).

${ }^{28}$ Sh. Michaelson, O. Ternyak, A. Hoffman, and Y. Lifshitz, Appl. Phys. Lett. 90, 031914 (2007).

${ }^{29}$ C. J. Tang, A. J. Neves, M. A. Neto, and A. J. S. Ferrnandes, Diamond Relat. Mater. 13, 769 (2004).

${ }^{30}$ C. J. Tang, M. A. Neto, M. J. Soares, A. J. S. Fernanders, A. J. Neves, and J. Grácio, Thin Solid Films 515, 3539 (2007).

${ }^{31}$ M. Alam and A. Lucero, Mater. Sci. Eng., B 27, 81 (1994). 